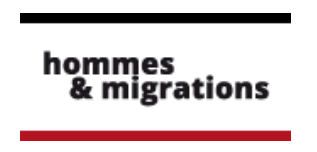

\section{Hommes \& migrations}

Revue française de référence sur les dynamiques

migratoires

1319 | 2017

Réfugiés et migrants au Liban

\title{
Quand la passion du cirque se décline en famille : l'Académie Fratellini ou la transmission d'une tradition italienne
}

Entretien avec Annie Fratellini

\section{Michela Slataper}

\section{OpenEdition}

\section{Journals}

Édition électronique

URL : http://journals.openedition.org/hommesmigrations/3999

DOI : ERREUR PDO dans /localdata/www-bin/Core/Core/Db/Db.class.php L.34 : SQLSTATE[HYO0O]

[2006] MySQL server has gone away

ISSN : 2262-3353

Éditeur

Musée national de l'histoire de l'immigration

Édition imprimée

Date de publication : 1 octobre 2017

Pagination : 155-157

ISBN : 978-2-919040-39-1

ISSN : 1142-852X

\section{Référence électronique}

Michela Slataper, «Quand la passion du cirque se décline en famille : I'Académie Fratellini ou la transmission d'une tradition italienne », Hommes \& migrations [En ligne], 1319 | 2017, mis en ligne le 01 octobre 2017, consulté le 07 janvier 2021. URL : http://journals.openedition.org/hommesmigrations/ 3999 ; DOI : https://doi.org/10.4000/hommesmigrations.3999 


\section{QUAND LA PASSION DU CIRQUE SE DÉCLINE EN FAMILLE :}

\section{L'ACADÉMIE FRATELLINI OU LA TRANSMISSION D'UNE TRADITION ITALIENNE}

Entretien avec VALÉRIE FRATELLINI, réalisé par MICHELA SLATAPER.

e cirque doit beaucoup à l'Italie et beaucoup Ldoivent les clowns à la Commedia dell'Arte. Les plus grands artistes de la première moitié du $X X^{e}$ siècle ont célébré les Fratellini, trois frères d'origine italienne qui ont révolutionné l'histoire des clowns en constituant, en 1910, le légendaire trio qui portait leur nom. La passion de ces trois artistes, généreux et infatigables travailleurs, se transmet aujourd'hui aux nouvelles générations au sein de l'Académie Fratellini, basée à La Plaine Saint-Denis. Dans le droit fil d'une tradition familiale qui a donné à l'univers du cirque plusieurs générations d'artistes, Valérie Fratellini est aujourd'hui la directrice pédagogique de l'Académie. Fille d'Annie Fratellini', fondatrice avec Pierre Etaix de l'école du cirque dont l'Académie est la continuité, elle revient sur une épopée circassienne qui plonge ses racines dans la péninsule italienne.

Hommes \& Migrations : Valérie Fratellini, vous venez d'une dynastie d'artistes de cirque dont vous pérennisez la tradition. Depuis combien de générations les Fratellini sont-ils liés au monde du cirque ?
Valérie Fratellini : Je compte cinq générations. Moi,j'appartiens à la cinquième. Tout a commencé à Florence avec Gustavo² dans la deuxième moitié du XIXe siècle. Son père voulait qu'il fasse des études de médecine, mais il a épousé une blanchisseuse (Giovanna Pilori qui, dans famille, est appelée « la nonna ») et il est parti sur les routes avec une troupe de saltimbanques.

Gustavo et Giovanna ont eu quatre enfants (à l'origine "les frères Fratellini » étaient, en effet, quatre) : Luigi, Paolo, Alberto et Francesco ${ }^{4}$, mais Luigi est décédé jeune, lors d'une tournée en Pologne. Après sa mort, Paolo, Alberto et Francesco ont créé le trio de clowns qui les a rendus légendaires dans le monde du cirque et des arts 5 . Moi, je descends de la branche de Paolo qui a eu six enfants, dont Victor, mon grand-père, le père de Annie.

Reconstituer la dynastie n'est pas facile, car il y a beaucoup de ramifications, dont une (celle de Francesco) en Amérique. J'ai encore une lointaine cousine qui vit à Moscou. De la branche de Paolo, il n'y a plus guère que moi et mon cousin Arnaud. En revanche, l'arrière-petit-fils de Francesco, 


\section{ITALIANITÉ}

qui s'appelle Francesco lui-même, poursuit avec succès sa carrière de clown. On m'en a dit beaucoup de bien mais je ne l'ai pas encore vu.

Alberto n'a eu qu'une fille, Louise, morte très jeune et sans laisser de descendance. Par contre, on doit à Alberto un livre de mémoires : Nous, les Fratellini, publié en 1955.

\section{H\&M : Tous les Fratellini ont été clowns. Comment se transmettait cette tradition au sein de la famille?}

V.F. : Au départ Gustavo était acrobate. Quand il n'a plus été capable d'exécuter un salto mortale ${ }^{6}$, il s'est maquillé et a commencé à faire rire les gens. Ils étaient tous acrobates et sont tous devenus clowns, passé un certain âge, parce qu'il fallait bien vivre, manger, continuer à travailler. Mais, comme on peut le voir sur les photographies qu'avait gardées l'arrière-grand-père Paolo, toutes les occasions étaient bonnes pour s'amuser : ils faisaient du cirque tout le temps. Et ils l'enseignaient tout naturellement aux enfants: les acrobaties, le maquillage, le jeu. Ils ne décidaient pas, c'était comme ça, " on faisait comme papa ». II n'y a qu'Alberto, je crois - le petit dernier de la famille - qui était le clown de la famille. Il n'a été que clown car il était fait pour cela, je le pense réellement. Pour les autres, il y a des photographies qui attestent qu'ils ont été, dans un premier temps, des acrobates.

\section{H\&M : D'où leur venait cette appétence pour le jeu, cette nature exubérante, d'après vous?}

V.F. : Ils jouaient avec tout, et ils ne s'arrêtaient jamais. Il y a dans cette attitude un côté Commedia dell'Arte? , tradition de théâtre qui est d'ailleurs à l'origine de l'art du cirque.

\section{H\&M : Une tradition bien italienne donc...}

V.F. : Complètement. Elle a failli s'éteindre avec Annie, et elle a été "sauvée » par un Français, Pierre Etaix ${ }^{8}$, qui a poussé Annie à reprendre le flambeau. Il lui a dit un jour: "Tu es une Fratellini, tu ne peux qu'être clown. "Ma mère, en revanche, se sentait comédienne et chanteuse. Dès son adolescence, elle n'a voulu qu'une chose : quitter sa famille. Elle avait été élevée à la dure par son père qui l'enfermait dans sa chambre pour qu'elle travaille la musique. Quand il entendait le métronome s'arrêter, il ouvrait la porte. II la surveillait. II fallait qu'elle apprenne le métier et elle a commencé à travailler et à gagner de l'argent dès l'âge de 13-14 ans. Tous ses cachets étaient remis à la famille. Annie voulait aller à l'école, étudier, être comme toutes ses amies... Puis il y avait la lourdeur de la promiscuité, dont je me souviens moi-même, ayant été élevée par mes grands-parents. Lors des repas familiaux, tous (enfants, petits-enfants, tantes) prenaient place à une grande table présidée par le grand-père. On pouvait commencer à manger seulement quand le patriarche était assis. Il portait un chapeau et un gilet et goûtait les pâtes que mes tantes avaient fabriquées le matin. Ensuite, il émettait un jugement; " C'est bon, c'est al dente... » ou «Ce n'est pas bon. » Une situation assez étonnante : même si Victor n'avait jamais vécu en Italie et le reste de la famille non plus, ils gardaient cette identité de famille patriarcale.

Annie avait de très bons souvenirs avec son grandpère et sa grand-mère, mais elle a fui la promiscuité et l'enfermement familial le jour de ses dixhuit ans. Elle a dû se marier pour partir, mais elle est partie.

\section{H\&M : Cette promiscuité ne venait-elle pas du métier ?}




\section{/// « Arbre généalogique de la Famille Fratellini. »}

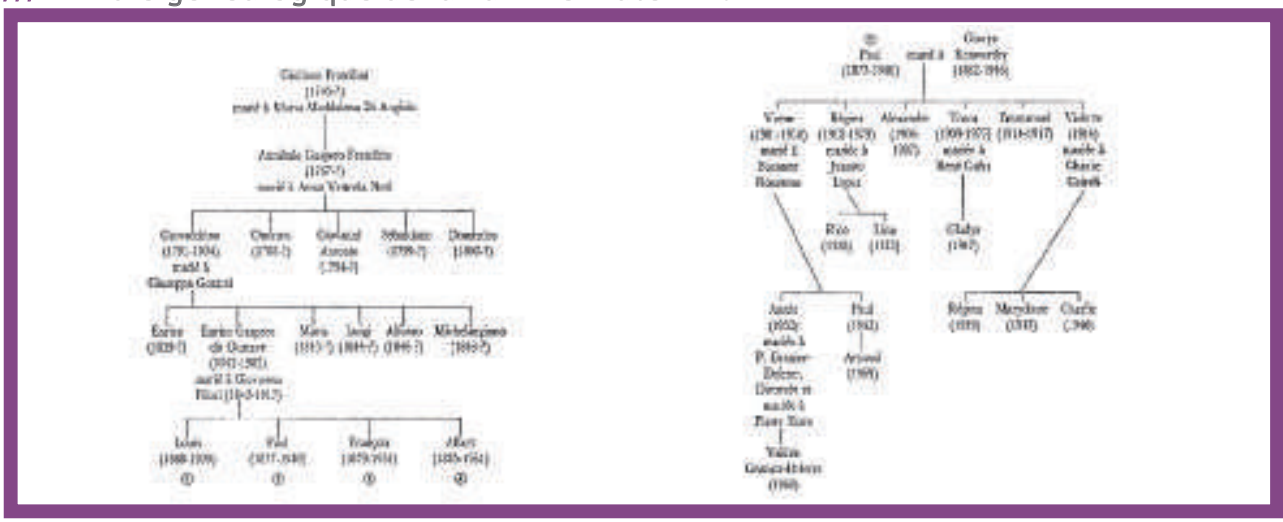

Source : Annie Fratellini, Destin de clown, Lyon, La Manufacture, 1989.

V.F. : Non, car il faut dire que, quand les Fratellini partaient en tournée, ils allaient à l'hôtel. Dans les cirques en dur, c'est comme au théâtre, il n'y a pas du tout la même ambiance qu'on trouve dans les cirques itinérants. Les Fratellini étaient des sédentaires. Ils ont vécu au Perreux-sur-Marne, mais aussi autour de Montmartre pour être près du Cirque Medrano et du Cirque d'Hiver où ils ont quand-même travaillé pendant plusieurs années ${ }^{9}$. Annie a juste fui le poids de cette famille nombreuse, entièrement engagée dans le monde du cirque. On lui disait: "Tu es une Fratellini, tu dois faire de l'acrobatie et de la danse, après on verra... " Il y avait une sorte de fierté pour la tradition familiale.

\section{H\&M : C'est donc Pierre Etaix, un cinéaste français amoureux du monde du cirque, qui a perçu la richesse qu'Annie détenait : une tradition familiale et culturelle qu'il fallait préserver.}

V.F. : Je crois qu'en réalité il avait envie de voir sa femme en artiste du cirque. Quand il en parlait, il avait des paillettes plein les yeux. Jusqu'à sa mort, il a nourri un amour passionné pour les Fratellini. II en rêvait et il l'a fait : il a construit Annie en clown et récupéré la valeur de cet art, donc effectivement : merci Pierre!

$H \& M$ : Ensemble, ils se sont donc engagés dans la transmission de l'art du cirque aux nouvelles générations. Peut-on appeler l'Académie Fratellini une « pépinière d'artistes circassiens $"$ ?

V.F. : Pas tout à fait. L'Académie est un lieu d'apprentissage. Nous sommes un Centre de formation des apprentis (CFA) où l'on apprend le métier du cirque. Les élèves (douze par an) passent une audition et, s'ils sont admis, apprennent en trois ans à être devant le public, à créer avec les metteurs en scène, à créer eux-mêmes. Le cirque est très ouvert : ils ont la chance d'apprendre ce qu'ils veulent et surtout de faire en sorte que le corps et l'esprit ne fassent qu'un. C'est ce qu'il y a de plus difficile dans le métier. Le cirque à mon avis vit avec son temps, il se renouvelle constamment. En créant l'école du cirque, Pierre et Annie ont démarré une histoire qui ne va jamais s'arrêter celle d'un art qui nous transmet de la poésie et des valeurs telles l'attention à l'autre, I'humilité, l'entraide et le courage. Le cirque est une grande école de vie. 